\title{
Experimental Evidence for Sign Reversal of the Hall Coefficient in Three-Dimensional Metamaterials
}

\author{
Christian Kern, ${ }^{1,2}$ Muamer Kadic, ${ }^{1,2}$ and Martin Wegener ${ }^{1,2}$ \\ ${ }^{1}$ Institute of Applied Physics, Karlsruhe Institute of Technology (KIT), 76128 Karlsruhe, Germany \\ ${ }^{2}$ Institute of Nanotechnology, Karlsruhe Institute of Technology (KIT), 76021 Karlsruhe, Germany
}

(Received 18 July 2016; published 4 January 2017)

\begin{abstract}
Effectively inverting the sign of material parameters is a striking possibility arising from the concept of metamaterials. Here, we show that the electrical properties of a $p$-type semiconductor can be mimicked by a metamaterial solely made of an $n$-type semiconductor. By fabricating and characterizing threedimensional simple-cubic microlattices composed of interlocked hollow semiconducting tori, we demonstrate that sign and magnitude of the effective metamaterial Hall coefficient can be adjusted via a tori separation parameter-in agreement with previous theoretical and numerical predictions.
\end{abstract}

DOI: 10.1103/PhysRevLett.118.016601

The properties of composites or rationally designed artificial materials called metamaterials are generally not in between those of their constituents; i.e., "one gets out more than one puts in" [1,2]. Unbounded behavior is possible, too. Sign reversal of an effective material parameter with respect to the constituents is a particularly striking case. The availability of positive and negative parameter values largely enhances our ability to mold waves and fluxes. Thus, sign reversals have contributed much to the excitement in the field of metamaterials. In the dynamic case, they are well known by now. Examples are the magnetic permeability [3-5], the refractive index [4,6,7], the mechanical compressibility $[8,9]$, the mass density [10-12], or both of the latter [13-16]. In all of these examples, the sign inversion arises from some sort of internal resonance. For frequencies above the corresponding eigenfrequency, the response exhibits a 180-degree phase shift, i.e., a reversal of sign. Clearly, this mechanism does not work in the stationary limit.

Moreover, for passive media in the stationary limit, due to the second law of thermodynamics, reversing the sign of transport coefficients like the electric conductivity, the heat conductivity, or the diffusivity is even fundamentally impossible. The Hall coefficient is a notable exception. It is directly connected to the off-diagonal elements of the electric conductivity tensor in the presence of a static magnetic field. In the simplest case, the Hall coefficient $A_{\mathrm{H}}$ is equal to the inverse of the charge density, i.e., $A_{H}=\rho^{-1}$. A few years ago, building upon earlier work [17-19], Marc Briane and Graeme W. Milton predicted theoretically that the sign of the isotropic Hall coefficient can be reversed in chainmail-like three-dimensional metamaterials [20]. Notably, art inspired science: Chainmail artist Dylon Whyte suggested to them the three-dimensional structure [21]. Sign reversal of the Hall coefficient in metamaterials is interesting because one can, for example, effectively mimic $p$-doped silicon when having only $n$-doped silicon available as a constituent. However, an experimental validation of this mind-boggling prediction has not been published so far.

In this Letter, we present experimental evidence for the predicted sign inversion of the effective Hall coefficient in chainmail-like metamaterials. We show that the sign as well as the magnitude of the Hall coefficient can be determined by a geometrical separation parameter while fixing the

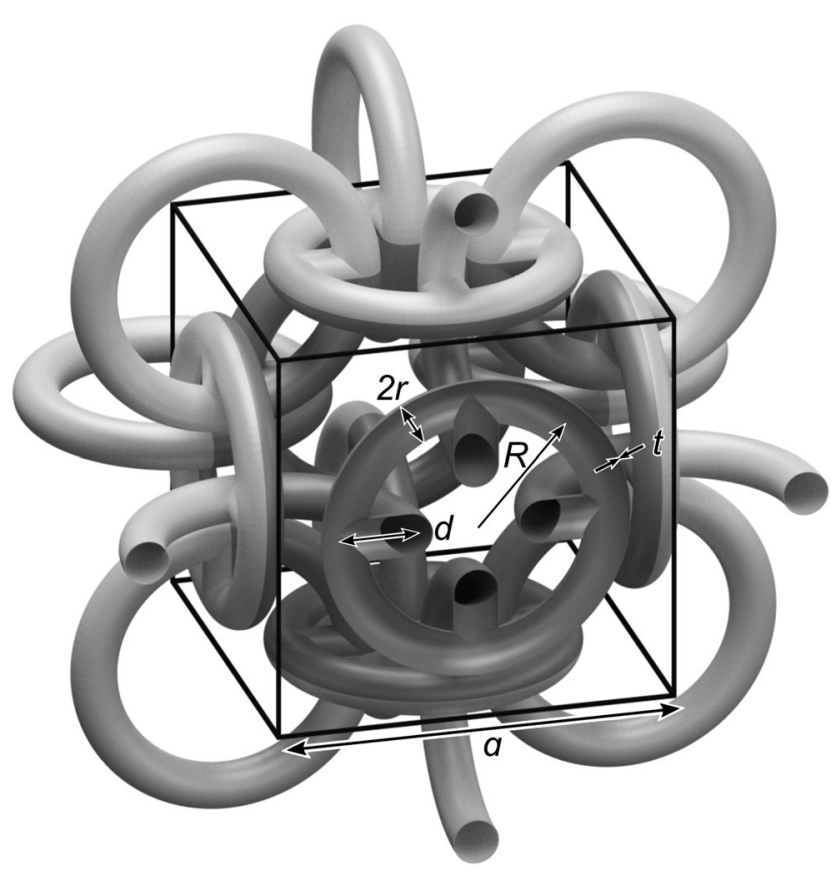

FIG. 1. Blueprint for the three-dimensional chainmail-like metamaterial discussed in this Letter. It is composed of a simple-cubic lattice of hollow tori. $a$ is the lattice constant, $R$ the torus radius, $r$ the wire radius, $t$ the film thickness, and $d$ the separation parameter. The latter can be positive or negative; the depicted configuration corresponds to $d<0$. 


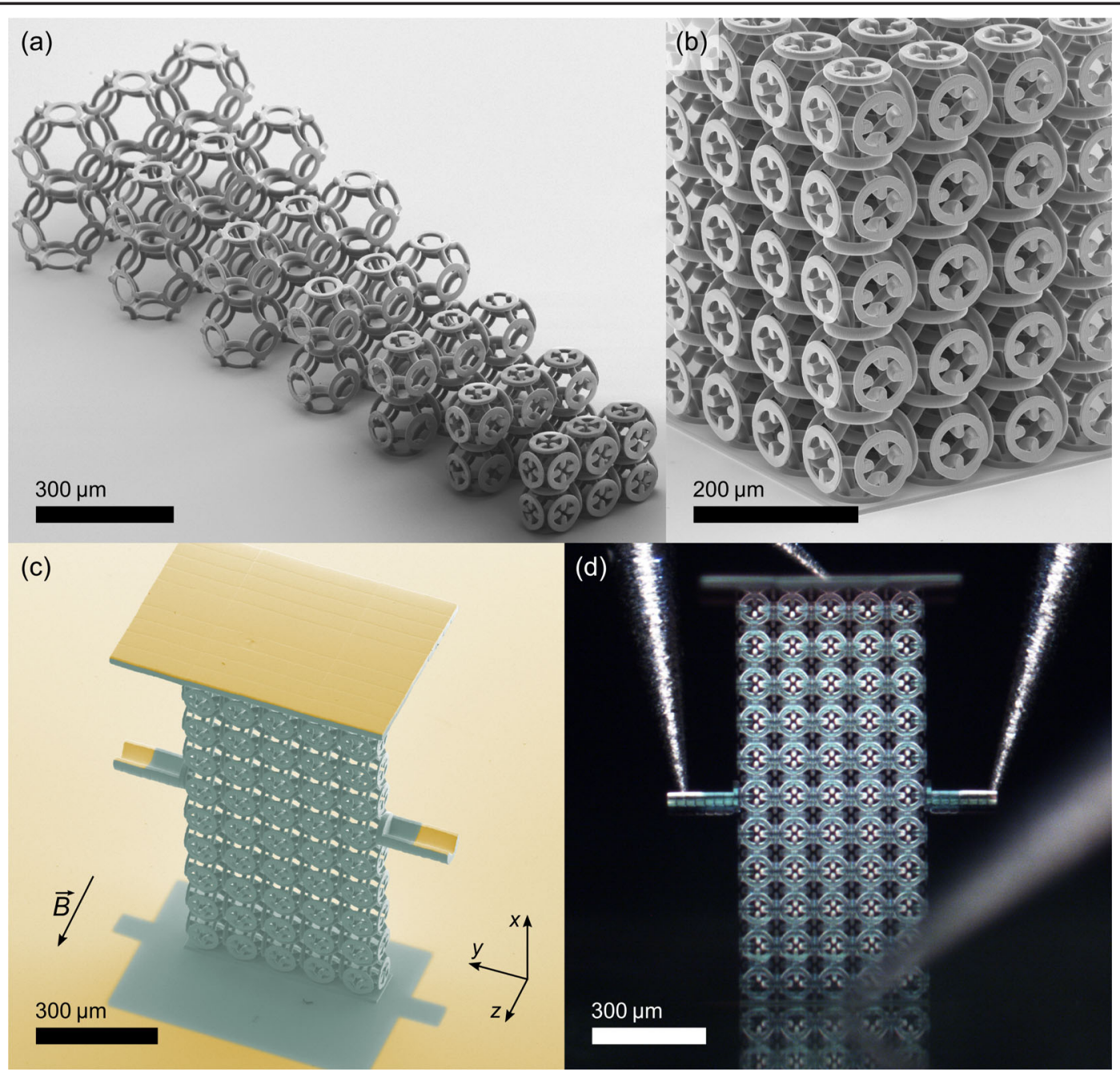

FIG. 2. Example images of fabricated three-dimensional metamaterial samples on glass substrates. (a)-(c) are oblique-view electron micrographs; (c) is colored for clarity, and (d) is an optical micrograph. (a) Sequence of polymer structures with different separation parameters $d$ (compare to Fig. 1). At fixed parameters $R, r$, and $t$, the lattice constant $a$ increases with increasing $d$ (from bottom right to top left). (b) Polymer structure coated with $n$-type $\mathrm{ZnO}$ with $4 \times 4 \times 5$ unit cells and $d<0$. (c) shows a Hall bar composed of $11 \times 5 \times 1$ unit cells. The roof on top casts a shadow during evaporation of the metal contacts onto the substrate and the two pick-up contacts. To ease the alignment of the tungsten contact needles, halfpipes have been fabricated on the left and right. (d) Structure as in (c) within the home-built electrical probe station. The four electrical contact probes are visible.

constituent material. The blueprint of the considered structure is illustrated in Fig. 1.

The samples discussed here have been fabricated in three steps. First, we write polymer structures by standard threedimensional dip-in galvo-scanner-based laser lithography (Photonics Professional GT, Nanoscribe $\mathrm{GmbH}$ ) using a commercial photoresist (IP-S, Nanoscribe $\mathrm{GmbH}$ ) on glass substrates coated with a thin layer of indium-tin-oxide. These polymer structures are insulating electrically and merely serve as scaffolds. In a second step, using conformal atomic-layer deposition (Savannah 100, Cambridge Nanotech, Inc.), these support structures are coated with a $t=185 \mathrm{~nm}$ thin film of $n$-type $\mathrm{ZnO}$. The films are grown at $150^{\circ} \mathrm{C}$ substrate temperature using diethylzinc and water as precursors. The film thickness has been determined by ellipsometry. We choose $\mathrm{n}-\mathrm{ZnO}$ solely because we have experience with its deposition from different experiments [22] and because very smooth films can be achieved. Many other semiconductor materials should be possible, too. Third, to obtain Ohmic contacts, we evaporate titanium and gold with thicknesses of 30 and $100 \mathrm{~nm}$, respectively, by using standard high-vacuum electron-beam evaporation. The polymer roof plate on top of each structure casts a shadow to protect the Hall bar. The Hall contacts stick out of the roof's shadow to the left and right. A gallery of images of fabricated samples is shown in Fig. 2.

The samples are contacted in a home-built room-temperature probe station by four tungsten needles, which are connected to independent three-dimensional translation stages. Using a home-built electronics, we can quickly 
check the resistance between any of the twelve possible pairs out of the four contacts. After proper alignment, all combinations exhibit an Ohmic behavior. The longitudinal resistance $R_{x}=U_{x} / I_{x}$ is typically around $2 \mathrm{k} \Omega$. The probe station also allows us to move a permanent magnet combined with an iron yoke up and down with respect to the fixed sample. The sign of the magnetic field can be changed by mechanically rotating the magnet assembly by $180^{\circ}$. In the "on" position, the magnetic induction is about one Tesla, precisely $B_{z}= \pm 0.83 \mathrm{~T}$. In the "off" position, it is $\pm 0.03 \mathrm{~T}$. These values have been measured at the sample location using an independent commercial Hall sensor (HGT-2010, Lake Shore Cryotronics, Inc.). In the Hall measurements, the current $I_{x}$ is imposed by a constantcurrent source (B2901A source-measurement unit, Keysight Technologies, Inc.).

As usual, the Hall voltage is inversely proportional to the sample thickness along the direction of the magnetic field vector. Large Hall voltages are desirable to obtain good signal-to-noise ratios. Thus, we have performed all actual measurements using Hall bars with only a single threedimensional lattice cell along the $z$-direction (see Fig. 2). Precisely, the two polymer tori in the unit cell parallel to the $x y$-plane are cut in half and then coated with $n$-type $\mathrm{ZnO}$.

An example of acquired raw data of the transverse voltage $U_{y}$ vs real time is shown in Fig. 3(a). The temporal peaks in the transverse voltage $U_{y}$ arise from Faraday induction into wire loops in our setup while the magnet is moving. Hence, the voltages are defined with respect to times at which the magnet is at rest (compare bottom panel, showing the independently measured $B_{z}$ vs time). The blue and red traces in Fig. 3(a) provide direct experimental evidence that the transverse voltage $U_{y}=U_{0}+U_{H}$ consists of a magnetic-field independent contribution (that we refer to as the offset voltage $U_{0}$ ) and a contribution that depends on the magnetic field $B_{z}$, i.e., the Hall voltage $U_{H}\left(B_{z}\right)$. Such offset voltages are well known: For example, they can result from asymmetries of the pick-up contacts or fabrication imperfections breaking the cubic symmetry of the microlattice. In our experiments, this sample-dependent offset voltage $U_{0} \propto U_{x}=R_{x} I_{x}$ is typically on the order of $1 \mathrm{mV}$ at $I_{x}=0.5 \mathrm{~mA}$, which is about a thousand times smaller than the voltage $U_{x}=R_{x} I_{x}$, yet it is still larger than the Hall voltage. The offset voltage does not depend on the applied magnetic field $B_{z}$. In contrast, the Hall voltage $U_{H}$ does depend on the sign and amplitude of the magnetic field. Thus, by changing the sign of the magnetic field, the asymmetry offset voltage can simply be subtracted. Figure 3(b) shows the extracted Hall voltage $U_{H}$ vs injection current $I_{x}$ for two different structures. As expected, we find a proportional behavior for all samples, allowing us to extract a Hall resistance $R_{H}=U_{H} / I_{x}$ for the peak magnetic induction of $\left|B_{z}\right|=0.83 \mathrm{~T}$.

The gallery of electron micrographs in Fig. 2 has already shown structures with different separation parameter $d$
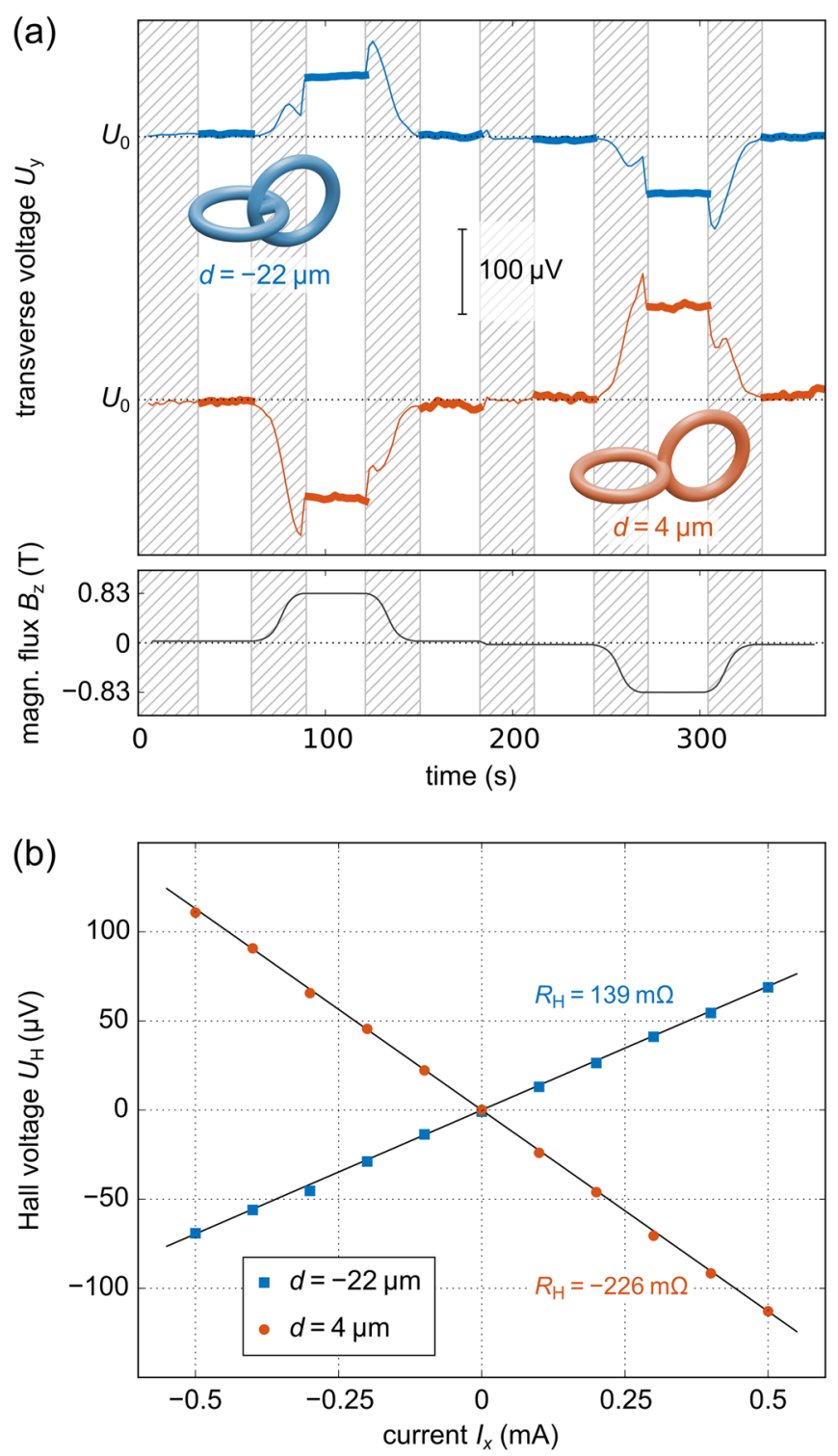

FIG. 3. (a) Measured transverse voltage $U_{y}$ versus real time. The two examples shown correspond to two Hall bars $[11 \times 5 \times 1$ unit cells, cf., Figs. $2(\mathrm{c})$ and $2(\mathrm{~d})]$ with different separation parameters, namely $d=-22 \mu \mathrm{m}$ (blue) and $d=$ $4 \mu \mathrm{m}$ (red). The magnet is moving in the shaded regions. Apart from the asymmetry offset voltage $U_{0}$, the voltage reverses sign upon reversing the sign of the magnetic induction $B_{z}$. The design parameters are $R=36 \mu \mathrm{m}$, $r=6 \mu \mathrm{m}, t=185 \mathrm{~nm}$, and $I_{x}=0.5 \mathrm{~mA}$. In the lower panel, the measured magnetic flux $B_{z}$ at the sample position vs time is shown. The measurement has been performed using a commercial Hall sensor. (b) Extracted Hall voltage (dots) vs injection current $I_{x}$ for the same samples. They exhibit different slopes of the Hall voltage $U_{H}$ vs $I_{x}$, i.e., effective Hall resistances $R_{H}$ of different sign. The effective Hall coefficient results as $A_{H}=R_{H} L_{z}(0.83 \mathrm{~T})^{-1}$, with the Hall bar thickness $L_{z}=a=4 R+2 d$. The straight lines are fits to the data. The underlying $n$-type $\mathrm{ZnO}$ constituent material has a negative Hall coefficient in bulk form. Thus, the case of $d=-22 \mu \mathrm{m}$ corresponds to a sign reversal of the Hall coefficient. 


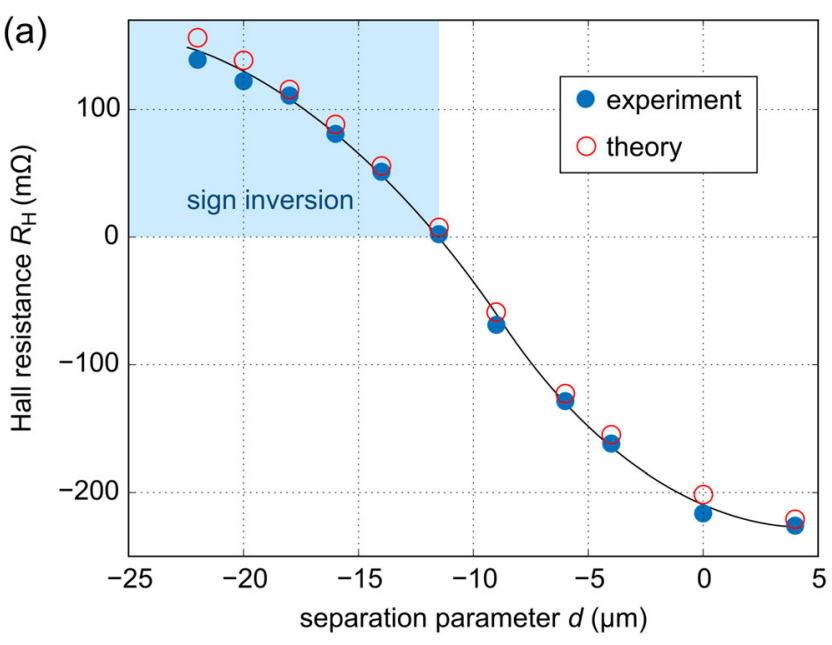

(b)

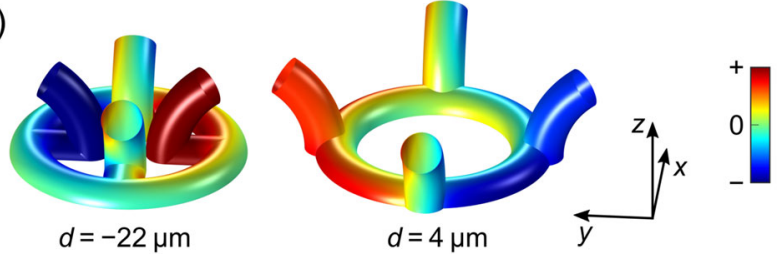

FIG. 4. (a) Hall resistance $R_{H}$ vs separation parameter $d$ as extracted from data as shown in Fig. 3(b). Each full blue dot corresponds to one sample and a measurement like in Fig. 3(b); the open red dots are calculated. The solid curve is a guide to the eye. Clearly, we find a sign reversal of the Hall resistance and hence of the Hall coefficient around $d=-12 \mu \mathrm{m}$. The design parameters are as in Fig. 3. (b) Corresponding calculated Hall potential landscape in a part of the central region of the Hall bar for two different values of $d$.

(also compare to Fig. 1). The behavior of the extracted Hall resistance $R_{H}$ vs $d$ is depicted in Fig. 4(a) for both positive and negative values of $d$. Each data point corresponds to a different sample, measured like in Fig. 3. We find that the Hall resistance in Fig. 4 and hence the Hall coefficient changes sign at around a separation parameter of $d=-12 \mu \mathrm{m}$.

In the numerical calculations also shown in Fig. 4(a), we use the commercial software package COMSOL Multiphysics to solve the stationary continuity equation $\vec{\nabla} \cdot(\stackrel{\leftrightarrow}{\sigma} \vec{\nabla} \phi)=0$ for the electrostatic potential $\phi=\phi(\vec{r})$. We use the usual form for the conductivity tensor $\stackrel{\leftrightarrow}{\sigma}=\stackrel{\leftrightarrow}{\sigma}(\vec{r})$ in the presence of a static homogeneous magnetic field $\vec{B}=\left(0,0, B_{z}\right)^{\top}$ along the $z$-axis within the linear regime [20,23],

$$
\stackrel{\leftrightarrow}{\sigma}=\sigma_{0}\left(\begin{array}{ccc}
1 & \tilde{B} & 0 \\
-\tilde{B} & 1 & 0 \\
0 & 0 & 1
\end{array}\right)
$$

$\tilde{B}=\sigma_{0} \rho^{-1} B_{z}$ is a dimensionless abbreviation, $\sigma_{0}=\sigma_{0}(\vec{r})$ is the piecewise constant zero-field conductivity, and $\rho=$ $\rho(\vec{r})$ is the piecewise constant charge density. The linear approximation is well justified for our conditions because $\tilde{B} \ll 1$ and $U_{H} / U_{x} \ll 1$. The conductivity $\sigma_{0}$ of the air voids and of the polymer is assumed to be 0 . Within the linear regime and for fixed $I_{x}$, the nonzero $\mathrm{ZnO}$ conductivity $\sigma_{0}$ does not enter the results and the $\mathrm{ZnO}$ charge density $\rho$ merely scales the Hall resistance in Fig. 4 according to $R_{H} \propto \rho^{-1}$. Thus, we have used $\rho$ as a fit parameter with $\rho=-2.6 \times 10^{6} \mathrm{C} \mathrm{m}^{-3}$. We consider a finite metamaterial Hall bar composed of $11 \times 5 \times 1$ unit cells. Because of fabrication limitations, the actual parameters are not quite identical to the quoted design parameters (see Fig. 2). Thus, we use the design parameters as above, except for $r=7.5 \mu \mathrm{m}$. This choice leads to excellent agreement between theory and experiment. In particular, the zero crossing at around $d=-12 \mu \mathrm{m}$ is well reproduced. From similar previous calculations [23] we can conclude that the investigated geometries exhibit only small effects related to the finite number of unit cells in all directions. The measured signatures can thus be interpreted as effective bulk effects.

To clarify the underlying physics, Fig. 4(b) visualizes the calculated Hall potential (false-color scale) for two characteristic values of $d$ and otherwise identical parameters. We define the Hall potential as the difference of the electrostatic potential $\phi(\vec{r})$ for $B_{z}=0.83 \mathrm{~T}$ and that for $B_{z}=0$. In this fashion, we subtract the overwhelmingly large but trivial linear potential ramp due to the applied voltage $U_{x}$. For clarity, only a part of the central region of the Hall bar is shown. Upon changing $d$, two aspects change. First, the Hall voltage is picked up differently by the tori parallel to the $y z$-plane. Second, the tori parallel to the $x z$-plane inject the electric current differently into the torus parallel to the $x y$-plane. As a result, the outer (inner) part of this torus is on nearly zero Hall potential (green) for $d=-22 \mu \mathrm{m}(d=+4 \mu \mathrm{m})$. Clearly, the two aspects are not independent. Their interplay leads to the sign reversal and shifts the zero crossing of $R_{H}$ away from $d=0$. Intuitively, for a straight hollow cylinder, the zero crossing would have to be at $d=0$ by symmetry. However, the curvature of the torus breaks this symmetry.

In conclusion, we have experimentally demonstrated the possibility of sign reversal of the static Hall coefficient in chainmail-like three-dimensional metamaterials. Intuitively, the sign reversal means that one can completely mimic the electrical properties of $p$-doped silicon by using microstructured $n$-doped silicon. Mathematically, one can tailor the sign and magnitude of the off-diagonal elements of the effective electric conductivity tensor in the plane normal to the magnetic-field vector. This freedom can be extended to all off-diagonal elements by going from cubic symmetry to lower symmetries $[24,25]$. In this case, an unusual Hall voltage parallel rather than orthogonal to the magnetic-field vector may arise. The findings of this work also have interesting possible implications for the dynamic Hall effect (or photon-drag effect), which is a second-order nonlinear optical process that can be nonzero in the presence of 
inversion symmetry. Herein, the incident electromagnetic wave replaces the applied static magnetic field and the applied static electric field. By using chainmail-like metamaterials, one could likewise control the sign and magnitude of second-harmonic generation or of the photon-drag current.

We thank Jurana Hetterich [Karlsruhe Institute of Technology (KIT)] for help with the ellipsometry, Michael Hetterich (KIT) for help in the early phase of the experiment, Martin Schumann and Andreas Wickberg (both KIT) for taking the scanning electron micrographs, and Michael Hippe, Frank Landhäuser, and Johann Westhauser (all KIT) for technical assistance. We acknowledge support by the Karlsruhe School of Optics \& Photonics (KSOP), by the Hector Fellow Academy, the KIT Nanostructure Service Laboratory, and by the Helmholtz Program Science and Technology of Nanosystems (STN).

[1] G. W. Milton, The Theory of Composites (Cambridge University Press, Cambridge, 2002).

[2] B. Banerjee, An Introduction to Metamaterials and Waves in Composites (Taylor \& Francis, London, 2011).

[3] J. B. Pendry, A. J. Holden, D. J. Robbins, and W. J. Stewart, IEEE Trans. Microwave Theory Tech. 47, 2075 (1999).

[4] D. R. Smith, W. J. Padilla, D. C. Vier, S. C. Nemat-Nasser, and S. Schultz, Phys. Rev. Lett. 84, 4184 (2000).

[5] J. Zhou, T. Koschny, M. Kafesaki, E. N. Economou, J. B. Pendry, and C. M. Soukoulis, Phys. Rev. Lett. 95, 223902 (2005).

[6] S. Zhang, W. Fan, N. C. Panoiu, K. J. Malloy, R. M. Osgood, and S. R. J. Brueck, Phys. Rev. Lett. 95, 137404 (2005).
[7] C. García-Meca, J. Hurtado, J. Martí, A. Martínez, W. Dickson, and A. V. Zayats, Phys. Rev. Lett. 106, 067402 (2011).

[8] Z. Liu, X. Zhang, Y. Mao, Y. Y. Zhu, Z. Yang, C. T. Chan, and P. Sheng, Science 289, 1734 (2000).

[9] M. Kadic, T. Bückmann, R. Schittny, and M. Wegener, Rep. Prog. Phys. 76, 126501 (2013).

[10] J. Mei, Z. Liu, W. Wen, and P. Sheng, Phys. Rev. Lett. 96, 024301 (2006).

[11] G. W. Milton, New J. Phys. 9, 359 (2007).

[12] Z. Yang, J. Mei, M. Yang, N. H. Chan, and P. Sheng, Phys. Rev. Lett. 101, 204301 (2008).

[13] Y. Ding, Z. Liu, C. Qiu, and J. Shi, Phys. Rev. Lett. 99, 093904 (2007).

[14] S. H. Lee, C. M. Park, Y. M. Seo, Z. G. Wang, and C. K. Kim, Phys. Rev. Lett. 104, 054301 (2010).

[15] Y. Wu, Y. Lai, and Z.-Q. Zhang, Phys. Rev. Lett. 107, 105506 (2011).

[16] S. A. Cummer, J. Christensen, and A. Alù, Nat. Rev. Mater. 1, 16001 (2016).

[17] G. Alessandrini and V. Nesi, Arch. Ration. Mech. Anal. 158, 155 (2001).

[18] M. Briane, G. W. Milton, and V. Nesi, Arch. Ration. Mech. Anal. 173, 133 (2004).

[19] M. Briane, D. Manceau, and G. W. Milton, J. Math. Anal. Appl. 339, 1468 (2008).

[20] M. Briane and G. W. Milton, Arch. Ration. Mech. Anal. 193, 715 (2009).

[21] G. W. Milton (private communication).

[22] A. Frölich and M. Wegener, Opt. Mater. Express 1, 883 (2011).

[23] M. Kadic, R. Schittny, T. Bückmann, C. Kern, and M. Wegener, Phys. Rev. X 5, 021030 (2015).

[24] M. Briane and G. W. Milton, SIAM J. Appl. Math. 70, 1810 (2010).

[25] C. Kern, M. Kadic, and M. Wegener, Appl. Phys. Lett. 107, 132103 (2015). 